\title{
An overview of cancer research in South African academic and research institutions, 2013 - 2014
}

\author{
J Moodley, ${ }^{1,2} \mathrm{PhD}$, MMed (Public Health Medicine), MB ChB; D C Stefan, ${ }^{3} \mathrm{MD}$, PhD; V Sewram, ${ }^{4,5} \mathrm{PhD}, \mathrm{MPH}, \mathrm{PhD}$; \\ P Ruff, ${ }^{6}$ FCP, MMed (Int Med), MB BCh; M Freeman, ${ }^{7,8}$ MA (Clin Psych); K Asante-Shongwe, ${ }^{9}$ LLB, BA (Liberal Arts) \\ ${ }^{1}$ Cancer Research Initiative, Faculty of Health Sciences, University of Cape Town, South Africa \\ ${ }^{2}$ Women's Health Research Unit, School of Public Health and Family Medicine, Faculty of Health Sciences, University of Cape Town, South Africa \\ ${ }^{3}$ South African Medical Research Council, Cape Town, South Africa \\ ${ }^{4}$ African Cancer Institute, Faculty of Medicine and Health Sciences, Stellenbosch University, Tygerberg, Cape Town, South Africa \\ ${ }^{5}$ Division of Community Health, Department of Interdisciplinary Health Sciences, Faculty of Medicine and Health Sciences, Stellenbosch \\ University, Tygerberg, Cape Town, South Africa \\ ${ }^{6}$ Division of Medical Oncology, Faculty of Health Sciences, University of the Witwatersrand, Johannesburg, South Africa \\ ${ }^{7}$ Directorate of Non-Communicable Diseases, National Department of Health, Pretoria, South Africa \\ ${ }^{8}$ Department of Psychology, Faculty of Humanities, University of the Witwatersrand, Johannesburg, South Africa \\ ${ }^{9}$ Steve Biko Centre for Bioethics, Faculty of Health Sciences, University of the Witwatersrand, Johannesburg, South Africa
}

Corresponding author: J Moodley (jennifer.moodley@uct.ac.za)

\begin{abstract}
Background and objectives. Cancer is emerging as a critical public health problem in South Africa (SA). Recognising the importance of research in addressing the cancer burden, the Ministerial Advisory Committee on the Prevention and Control of Cancer (MACC) research working group undertook a review of the current cancer research landscape in SA and related this to the cancer burden.

Methods. Academic and research institutions in SA were contacted to provide information on the titles of all current and recently completed (2013/2014) cancer research projects. Three MACC research working group members used the project titles to independently classify the projects by type of research (basic, clinical and public health - projects could be classified in more than one category) and disease site. A more detailed classification of projects addressing the five most common cancers diagnosed in males and females in SA was conducted using an adapted Common Scientific Outline (CSO) categorisation.

Results. Information was available on 556 cancer research projects. Overall, 301 projects were classified as clinical, 254 as basic science and 71 as public health research. The most common cancers being researched were cancers of the breast ( $n=95$ projects) and cervix ( $n=43$ ), leukaemia $(n=36)$, non-Hodgkin's lymphoma $(n=35)$ and lung cancer $(n=23)$. Classification of the five most common cancers in males and females in SA, using the adapted CSO categories, showed that the majority of projects related to treatment, with relatively few projects on prevention, survivorship and patient perspectives.

Conclusion. Our findings established that there is a dearth of public health cancer research in SA.
\end{abstract}

S Afr Med J 2016;106(6):607-610. DOI:10.7196/SAMJ.2016.v106i6.10314

Cancer, a complex disease that places a significant burden on individuals, families, health services and society, is emerging as a critical public health problem in South Africa (SA) ${ }^{[1]}$ Based on the most recent South African National Cancer Registry data, 1 in 9 women and 1 in 8 men are at risk of developing cancer in their lifetime. ${ }^{[2]}$ The most commonly diagnosed cancers among women are those of the breast, cervix, colorectum and uterus and Kaposi's sarcoma, which together account for half of all female cancers. Among men the commonest cancers are those of the prostate, lung, colorectum and oesophagus and Kaposi's sarcoma, which together total $36 \%$ of all cancers in men. In SA, as elsewhere in Africa, the majority of patients present with late-stage disease, which is associated with poor outcomes. ${ }^{[3,4]}$ Worryingly, the proportion of all deaths attributed to cancer in SA has increased steadily over the past few years, from $7.3 \%$ in 2011 to $7.8 \%$ in 2012 and $8.3 \%$ in $2013 .{ }^{[5]}$ Of additional concern is the forecast that the number of new cancer cases is expected to increase by $46 \%$ by $2030 .{ }^{[6]}$ Unless addressed, the rising burden of cancer could counteract the gains made from the stabilising communicable disease rates in SA and increasing life expectancy. ${ }^{[7]}$ Research directed at understanding the epidemiology and distinct features of cancer in SA, such as factors associated with late stage at presentation and cost-effective early detection strategies, is crucial in addressing the cancer burden.
Cognisant of the growing burden of cancer in SA, the Minister of Health, Dr Aaron Motsoaledi, approved the establishment of the Ministerial Advisory Committee on the Prevention and Control of Cancer (MACC) in accordance with section 91(1) of the National Health Act 61 of 2003. ${ }^{[8]}$ The purpose of the MACC is to advise the Minister on all matters related to national cancer prevention and control. Recognising the importance of research in identifying, prioritising and addressing the cancer burden, the Committee tasked its research working group to review the current cancer research landscape in SA.

\section{Methods}

A cross-sectional survey was conducted between September 2014 and June 2015. The National Department of Health, through the office of the Director-General of Health, formally contacted 29 institutions (all 23 higher academic institutions and six institutions either conducting or funding health research in SA) via email requesting information on the titles of all current (2014) and recently completed (2013/2014) cancer research projects. Three reminder emails were subsequently sent to non-responding institutions. Information was entered into an Excel database and duplicate projects were removed. Three members of the MACC research working group used the project titles to 
independently classify research projects by: (i) disease site using the International Cancer Research Partnership classification; ${ }^{[9]}$ and (ii) scientific domain (basic, clinical and public health research). Project titles suggesting that the research was aimed at understanding fundamental biological systems were classified as basic science research; those suggesting that the research was conducted with human subjects or on material of human origin such as tissues, and for which it was likely that an investigator directly interacted with human subjects, were classified as clinical research; and research conducted at a population or organisational level, including health system research, environmental and occupational research or patient perspectives, was classified as public health research. Projects could be classified into more than one scientific domain. A more detailed analysis was conducted for the five most common male and female cancers diagnosed in SA, using an adaption of the Common Scientific Outline (CSO) classification system. ${ }^{[10]}$ The CSO classification is organised around six broad areas of scientific interest in cancer research, viz.: (i) biology; (ii) aetiology; (iii) prevention; (iv) early detection, diagnosis, and prognosis; $(v)$ treatment; and (vi) cancer control, survivorship, and outcomes research, and is used to discuss, compare and present cancer research portfolios. For our analysis we expanded the CSO categories into nine categories to provide more detail. The expanded adapted CSO categories were: (i) biology; (ii) aetiology; (iii) prevention; (iv) early detection and diagnosis; $(v)$ clinical prognosis/progression; (vi) treatment; (vii) clinical audits; (viii) survivorship; and (ix) patient perspectives. Research activity in the five most commonly diagnosed male and female cancers in SA was assessed. The most common cancers were identified using cancer incidence data from the SA National Cancer Registry. ${ }^{[2]}$

\section{Results}

Fifteen of the 29 institutions (52\%) responded directly, and information on an additional six institutions was obtained indirectly through responses from three SA organisations that fund cancer research, viz. the South African Medical Research Council, the National Research Foundation and the Cancer Association of South Africa. In total, these institutions provided information on 757 projects. Projects that were completed before $2013(n=110)$, duplicate projects submitted by both research funding agencies and recipient institutions $(n=38)$ and nonresearch projects $(n=53)$ were removed, leaving a total of 556 projects for analysis.
Scientific domain and disease site Overall, 301 projects were classified as clinical, 254 as basic science and 71 as public health research (Fig. 1). The majority of projects $(n=392)$ focused on a single disease site, 18 projects addressed multiple sites

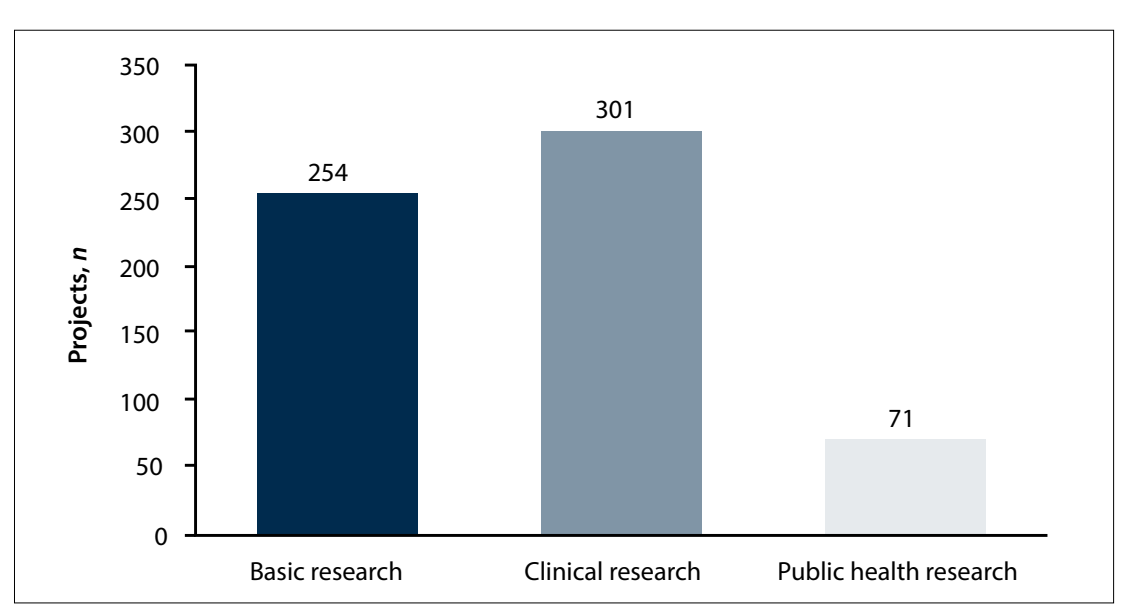

Fig. 1. Cancer projects classified by type of research domain (note: research projects could be categorised in more than one research domain).

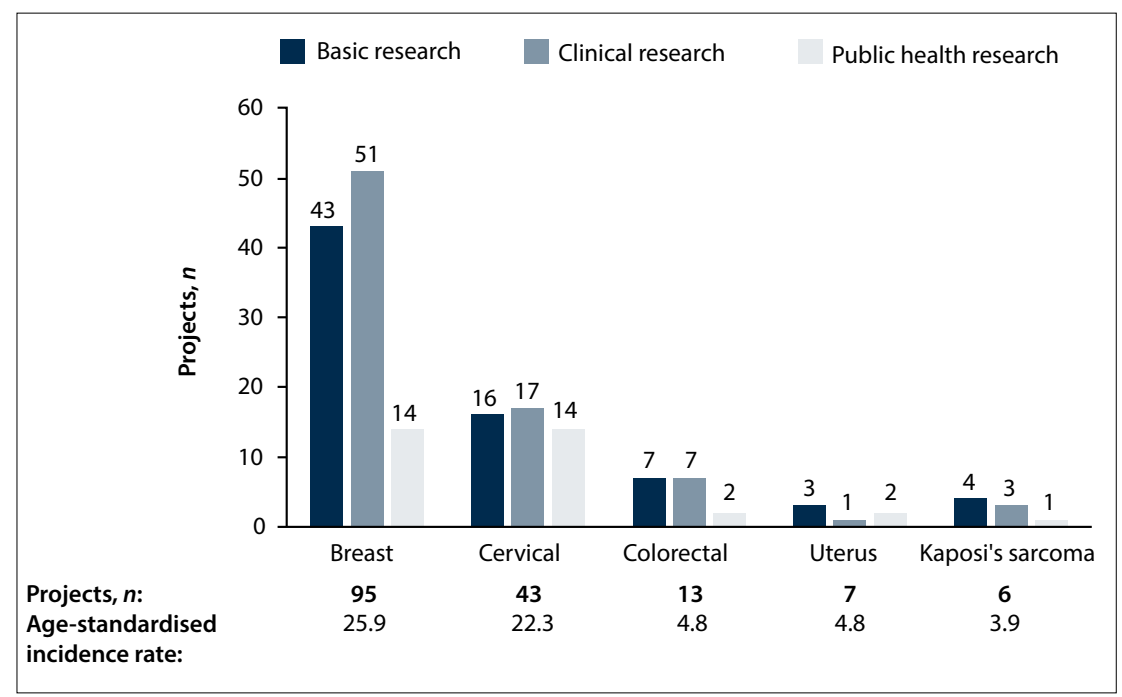

Fig. 2. Projects addressing the most commonly diagnosed female cancers in SA (note: research projects could be categorised in more than one research domain).

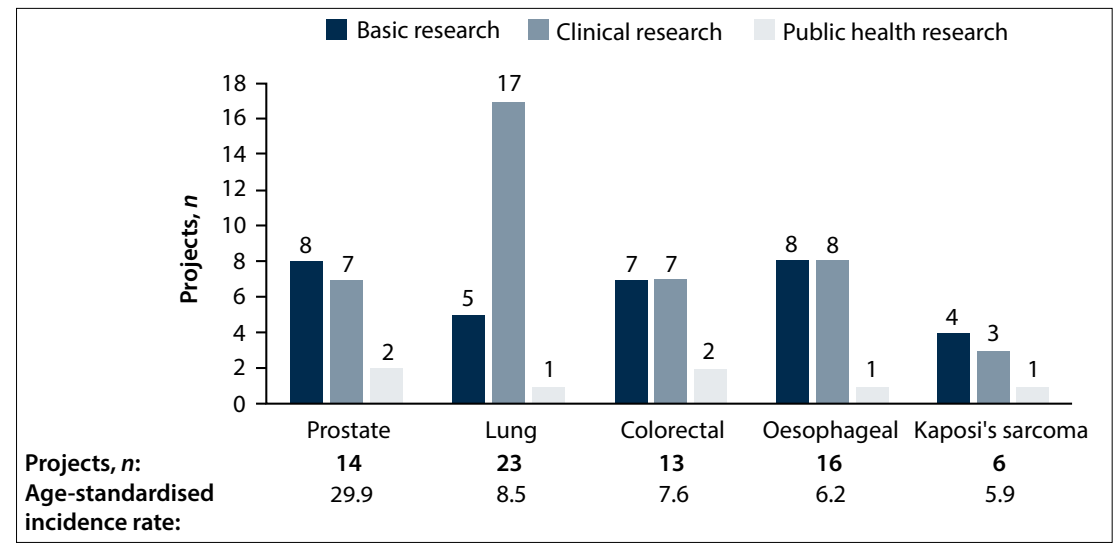

Fig. 3. Projects addressing the most commonly diagnosed male cancers in SA (note: research projects could be categorised in more than one research domain). 
Table 1. CSO classification for research projects addressing the five most commonly diagnosed cancers in SA

\begin{tabular}{|c|c|c|c|c|c|c|c|c|c|c|}
\hline & Biology & Aetiology & Prevention & $\begin{array}{l}\text { Early } \\
\text { detection } \\
\text { and } \\
\text { diagnosis }\end{array}$ & $\begin{array}{l}\text { Clinical } \\
\text { prognosis/ } \\
\text { progression/ } \\
\text { staging }\end{array}$ & Treatment & $\begin{array}{l}\text { Patient } \\
\text { profiles, } \\
\text { clinical audits } \\
\text { and review }\end{array}$ & Survivorship & $\begin{array}{l}\text { Patient } \\
\text { perspectives }\end{array}$ & Total \\
\hline \multicolumn{11}{|l|}{$\begin{array}{l}\text { Top five cancers } \\
\text { in females }\end{array}$} \\
\hline Breast & 12 & 18 & 2 & 9 & 14 & 35 & 3 & 2 & 0 & 95 \\
\hline Cervical & 5 & 4 & 13 & 2 & 6 & 8 & 3 & 0 & 1 & $42^{*}$ \\
\hline Colorectal & 3 & 2 & 0 & 0 & 0 & 3 & 4 & 0 & 0 & $12^{*}$ \\
\hline Uterus & 1 & 0 & 0 & 2 & 0 & 0 & 1 & 0 & 3 & 7 \\
\hline $\begin{array}{l}\text { Kaposi's } \\
\text { sarcoma }\end{array}$ & 2 & 1 & 0 & 0 & 2 & 0 & 1 & 0 & 0 & 6 \\
\hline Total & 23 & 25 & 15 & 13 & 22 & 46 & 12 & 2 & 4 & 162 \\
\hline \multicolumn{11}{|l|}{$\begin{array}{l}\text { Top five cancers } \\
\text { in males }\end{array}$} \\
\hline Prostate & 1 & 0 & 0 & 4 & 0 & 8 & 0 & 0 & 1 & 14 \\
\hline Lung & 1 & 2 & 1 & 1 & 1 & 16 & 0 & 0 & 1 & 23 \\
\hline Colorectal & 3 & 2 & 0 & 0 & 0 & 3 & 4 & 0 & 0 & $12^{*}$ \\
\hline Oesophageal & 4 & 4 & 0 & 0 & 2 & 6 & 0 & 0 & 0 & 16 \\
\hline $\begin{array}{l}\text { Kaposi's } \\
\text { sarcoma }\end{array}$ & 2 & 1 & 0 & 0 & 2 & 0 & 1 & 0 & 0 & 6 \\
\hline Total & 11 & 9 & 1 & 5 & 5 & 33 & 5 & 0 & 2 & 71 \\
\hline
\end{tabular}

cancer projects, with the vast majority $(n=24)$ classified as addressing clinical research. The majority of site-specific paediatric cancer research projects were on leukaemia $(n=4)$ and lymphomas $(n=4)$. The 556 projects included 95 clinical trials, with 64 being phase III clinical trials. The majority of clinical trials were related to breast cancer $(n=22)$, leukaemia $(n=15)$ and lung cancer $(n=14)$. There were no costing or cost-effectiveness studies.

\section{Research on the most common cancers diagnosed in SA}

A total of 164 projects addressed the five most common cancers diagnosed in women in SA (Fig. 2). Overall, clinical research was the most common type of research being conducted and public health research the least common. In contrast, for cervical cancer there were a similar number of research projects across all three scientific domains (i.e. basic science, clinical and public health). Classification using the adapted CSO categories (Table 1) showed that the majority of projects were related to treatment, with relatively few projects on survivorship and patient behaviour.

A total of 72 projects addressed the five most common cancers in males (Fig. 3). The majority of the projects were related to clinical research $(n=42)$, with relatively few $(n=7)$ addressing public health issues. Projects on lung cancer dominated, and according to CSO categorisation (Table 1) the majority of these were treatment-related projects. There were no projects being conducted on survivorship, and there was one project on prevention of cancer and two projects addressing patient perspectives.

\section{Discussion}

In an effort to understand whether current research addresses the burden of cancer in SA, academic and research organisations in the country were contacted for information on current and recently completed cancer research. Our findings established that there is a dearth of public health research. In a resourcelimited setting such as SA, public health cancer research offers many opportunities to address the cancer burden. For example, research that identifies reasons for late presentation, supports early diagnosis, identifies cost-effective management, improves the efficiency and effectiveness of cancer management and improves the quality of life of individuals with cancer will be particularly useful in informing local cancer policies and guidelines. It is of concern that there were no costing or cost-effectiveness cancer research projects. However, it is possible that desk-based economic studies that did not require ethical approval could inadvertently have been excluded from the list of projects submitted by institutions. In the SA context, economic studies could be invaluable in guiding diagnosis, treatment and prevention options as well as decisions on resource allocation. Public health cancer research could be limited as a result of lack of requisite research skills and/or limited funding for such research. This needs further investigation. Previous reports have highlighted the mismatch between the disease burden in developing countries and the human capacity to generate relevant new knowledge, ${ }^{[11,12]}$ and there have been calls to strengthen the health research capacity in the country. It is important that initiatives aimed at increasing research capacity are directed at priority health problems such as cancer, and that the gap in public health cancer research is addressed.

Breast cancer is the most common cancer diagnosed in women in SA, with an age-standardised incidence rate of 27 per 100000 ; however, in several regions in the country cervical cancer remains the most common cancer. ${ }^{[2]}$ It is encouraging that current research on female cancers focuses on the two most common cancers 
diagnosed in the country, viz. breast and cervical cancer. Further research on prevention, patient perspectives and survivorship is required. Overall, current research is skewed towards cancers most common in females as opposed to those most common in males. Relative to the burden of cancer in SA, limited research is being done on prostate cancer, colorectal cancer and Kaposi's sarcoma.

\section{Study limitations}

There were several limitations in our approach to determining current cancer research, and the results need to be interpreted with this in mind. Firstly, only 52\% of institutions contacted responded. However, the institutions in SA known to have a high research influence did provide information on their cancer research portfolios. ${ }^{[13]}$ Although each project was classified by more than one team member, information on project aims and objectives was unavailable, so classification by scientific domain and the expanded $\mathrm{CSO}$ categories was challenging and subject to misclassification. This review did not assess the quality of the research or review past cancer research. Further discussions on the challenges faced by local cancer researchers and funding agencies, the influence of local research on policies and guidelines and the extent to which research addresses the specific needs of the country are required to understand the SA cancer research landscape fully. The SA Medical Research Council is well placed to facilitate such discussions and future assessments.

\section{Conclusion}

Research is key to addressing the cancer burden in SA. This review highlighted the need for a cancer research registry that will provide ongoing information on the state of cancer research in the country, enable assessment of the relevance of such research, and assist in identifying research and funding priorities.

Acknowledgements. The authors thank the members of the MACC, Ms Sandhya Singh and Ms Bilqees Sayed from the National Department of Health, the Office of the Director-General of Health, and Ms Vedantha Singh from the Cancer Research Initiative, Faculty of Health Sciences, University of Cape Town. We are grateful to the institutions that provided information on current and recently completed cancer research.

\section{References}

1. Stefan DC. Why is cancer not a priority in South Africa? S Afr Med J 2015;105(2):103-104. DOI:10.7196/samj.9301

2. National Cancer Registry. Cancer in South Africa 2010. 2015. http://www.nioh.ac.za (accessed 5 November 2015).

3. Sylla B, Wild C. Cancer burden in Africa in 2030: Invest today and save tomorrow. Afr J Cancer 2012;4(1):1-2. DOI:10.1007/s12558-012-0199-4

4. Somdyala NI, Bradshaw D, Gelderblom W. Cancer Incidence in Selected Municipalities of the Eastern Cape Province, 2003-2007. Eastern Cape Province Cancer Registry Technical Report. Cape Town: South African Medical Research Council, 2013.

5. Statistics South Africa. Mortality and causes of death in South Africa, 2011: Findings from death notification. 2012. http://www.statssa.gov.za (accessed 8 April 2015).

6. International Agency for Research on Cancer. Globocan 2012: Estimated cancer incidence, mortality and prevalence worldwide in 2012. 2013. http://globocan.iarc.fr (accessed 15 April 2015).

7. Bradshaw D, Dorrington R, Laubscher R. Rapid Mortality Surveillance Report 2011. Cape Town: Bradshaw D, Dorrington R, Laubscher R. Rap
South African Medical Research Council, 2012 .

8. South African Parliament. National Health Act No. 61 of 2003. Pretoria: Government Printer, 2003. 8. South African Parliament. National Health Act No. 61 of 2003. Pretoria: Government Printer, 2003.
9. International Cancer Research Partnership. ICRP Cancer Type List. 2011. https://www.icrpartnership.

0. International Cancer Research Partnership. Common Scientific Outline. 2012. https://www. icrpartnership.org/CSO.cfm (accessed 26 August 2014).

11. Isjellmuiden C, Marais D, Becerra-Posada F, Ghannem H. Africa’s neglected area of human resources for health research - the way forward. S Afr Med J 2012;102(4):228-233.

2. Academy of Science of South Africa. Consensus Report on Revitalising Clinical Research in South Africa. Pretoria: ASSAf, 2009.

13. Reuters T. Times Higher Education World University Rankings 2014-2015. 2015. https://www. timeshighereducation.co.uk (accessed 15 August 2015).

Accepted 23 November 2015 